\title{
Analyse de la végétation aquatique fixée des cours d'eau lorrains en relation avec les paramètres d'environnement
}

\author{
N. Grasmück1 \\ J. Haury2 \\ L. Léglize 1 \\ S. Muller ${ }^{1}$
}

Mots clés : Macrophytes, eaux courantes, méthodologie, biotypologie, chimie de l'eau, géologie, morphométrie.

Les proliférations de macrophytes atteignent désormais les eaux courantes et entrainent de nombreuses nuisances. La compréhension de ce phénomène nécessite une étude approfondie des peuplements végétaux en place et des paramètres d'environnement qui les conditionnent, afin de déterminer les phytocénoses de référence et leurs séquences de dégradation. Lors de cette étude, vingt et un cours d'eau, situés en tête de bassin versant et représentatifs de la diversité géologique de la Lorraine, ont été prospectés. Le choix des stations d'étude a été réalisé en fonction d'un découpage abiotique, définissant des zones de cours d'eau homogènes au niveau de la géologie, la pente et la largeur. La pertinence de l'utilisation de la géologie (classes de perméabilité) comme premier critère hiérarchique de classification des cours d'eau est confirmée par les résultats de cette étude. Les analyses chimiques et les relevés morphométriques effectués sur chaque station d'étude ont conduit à individualiser quatre grands types de cours d'eau (sur granites, sur grès, sur calcaires et sur marnes), dont la végétation aquatique a été inventoriée. Des successions végétales typiques en fonction de la position amont-aval ont été observées. Les notions de milieux naturellement fragiles et d'espèces "à risque de prolifération " ont été affinées. Cette étude constitue la base d'une typologie des cours d'eau par la végétation aquatique.

\section{An analysis of the aquatic vegetation of streams of Lorraine in relation to environmental variables}

Keywords : Macrophytes, running water, methodology, biotypology, water chemistry, geology, morphometry.

Macrophyte proliferation occurs in streams and can produce many nuisances. Comprehension of this phenomenon requires a detailed study of the macrophyte populations and the environmental variables affecting them, so that the phytocoenoses can be described in relation to their stages of breakdown. During this study, twenty-one streams have been examined, all being situated at the head of the basin and representing the diverse geology of Lorraine. The study sites were chosen in relation to abiotic cut-off points defined by zones of streams that were homogeneous in terms of their geology, gradient and size. The relevance of using geology (permeability classes) as the first hierarchic criterion in the stream classification was confirmed by the results of this study. Chemical analyses and morphometric surveys at each study site identified four major types of stream (on granite, sandstone, limestone and marl), on which the aquatic vegetation has been surveyed. Some typical vegetation successions have been observed as a function of their position from source to river mouth. Concepts of naturally sensitive environments and species « of proliferation risk» have been improved. This study forms the basis for a typology of streams, using the aquatic vegetation.

\section{Introduction}

Les proliférations de végétaux aquatiques fixés ne touchent plus seulement les milieux d'eau douce stagnante. Ils atteignent désormais également les eaux

\footnotetext{
1. Centre de Recherches Ecologiques de l'Université de Metz, 1 Rue des Récollets, B.P. 4116, 57040 Metz Cedex 01, France.

2. ENSA Botanique et INRA Ecologie hydrobiologique, 65 Route de St Brieuc, 35042 Rennes Cedex, France.

Texte d'une communication effectuée dans le cadre du Colloque - Limmologie appliquée et application de la Limnologie Besançon, Franche-Comté, 16-19 Novembre 1992.
}

courantes et y entraînent de nombreuses nuisances : une baisse de la qualité de l'eau (modification des teneurs en oxygène dissous, élévation du $\mathrm{pH}$, etc...), des risques de colmatage des prises d'eau en cas d'entraînement, des perturbations d'écoulement en étiage et un aspect visuel inesthétique.

Ces dommages ont un coût qui se chiffre en millions de francs pour les gestionnaires du milieu tels que les Agences de l'Eau, par exemple : détarage des stations de jaugeage : 0,6 million de $F$ /an ou surcoût 
de la fabrication d'eau potable : 2,6 millions de F/an (Agence de l'Eau Seine-Normandie 1989).

Si le point de départ de ce phénomène réside sans aucun doute dans l'augmentation du niveau trophique du milieu, d'autres paramètres naturels (débits d'étiage, éclairement important...) ou d'origine anthropique (rejets domestiques, agricoles ou industriels, réaménagements drastiques, baisses de débits) viennent ensuite fragiliser cet équilibre.

Les objectifs de cette étude sont d'approfondir la connaissance de la distribution des végétaux aquatiques macrophytiques dans les cours d'eau de Lorraine. Il est en effet nécessaire de bien connaître les phytocénoses de référence et leur évolution amontaval ainsi que leurs séquences de dégradation avant de juger s'il y a déséquilibre et proliférations.

Une analyse fine des paramètres susceptibles d'influer sur les peuplements végétaux apparaît donc indispensable.

Cet article présente les résultats d'une campagne d'acquisition de données où seule la végétation aquatique est prise en compte (hydrophytes et hélophytes).

\section{Milieux et méthodes d'étude}

L'acquisition des données a été réalisée selon une proposition méthodologique définie lors d'un programme d'étude inter-agences intitulé : « Etude des végétaux fixés en relation avec la qualité du milieu » (Léglize et al. 1990).

Les facteurs essentiels déterminant l'installation et de développement des végétaux aquatiques y sont décrits et leur acquisition formalisée selon un cadre hiérarchisé (Tableau 1).

\subsection{Cadre géographique}

17 cours d'eau et 4 rus ont été retenus (Fig. 1 et Tableau 2) en tenant compte de l'hydrogéologie (Agence de l'Eau Rhin-Meuse 1982), de la qualité de l'eau (Agence de l'Eau Rhin-Meuse 1989) et de l'ordre de drainage, également appelé règle des confluences (Strahler 1964).

Ce choix constitue donc un échantillonnage représentatif de la diversité géologique du bassin RhinMeuse. Il permet également d'étudier plusieurs cours d'eau (au minimum deux) appartenant au même type de substrat.
Tableau 1. Présentation schématique du cadre hiérarchisé d'étude des végétaux aquatiques en cours d'eau (d'après Univ. de Metz, 1991).

Table 1. Simplified introduction to the classification method for environmental parameters in relation to macrophytic growth in rivers (according to Univ. de Metz, 1991).
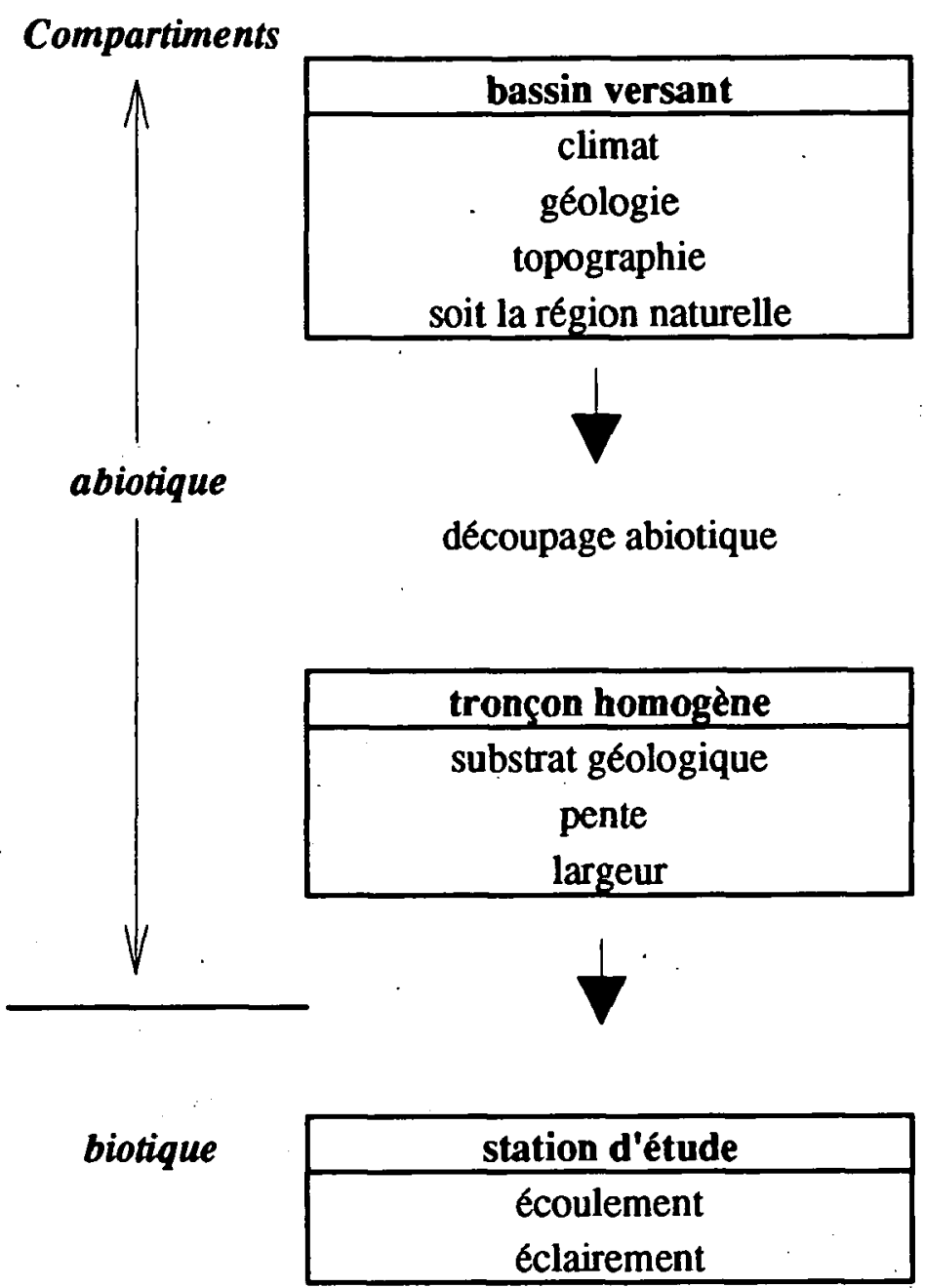

inventaire biocénoses

\subsection{Découpage des cours d'eau - Choix des stations}

Chaque cours d'eau a fait l'objet d'un découpage en segments homogènes pour, d'une part, la région naturelle et la perméabilité (Zumstein et al., 1989) et d'autre part, le dimensionnement (pente, calculée d'après le profil longitudinal et largeur entre berges, mesurée sur le terrain).

A ces facteurs naturels viennent s'ajouter des activités anthropiques plus ou moins perturbatrices, qui sont identifiées. Chaque cours d'eau étudié se subdivise ainsi en une succession de tronçons abiotiques.

Le choix des stations est effectué sur le terrain. Au sein d'un tronçon abiotique on choisit une ou quelques stations végétales représentatives de celui-ci. 

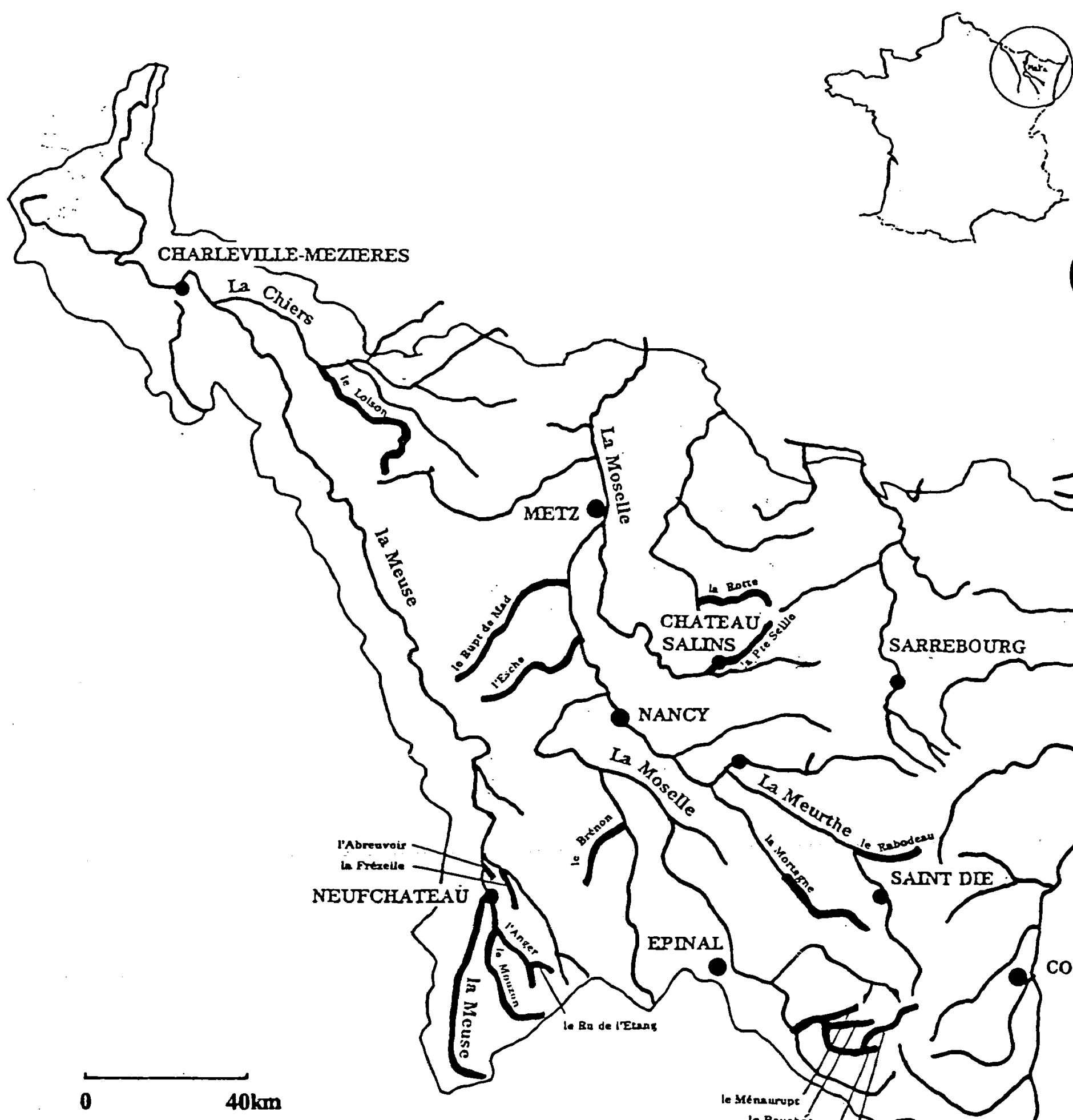

(A)

$40 \mathrm{~km}$
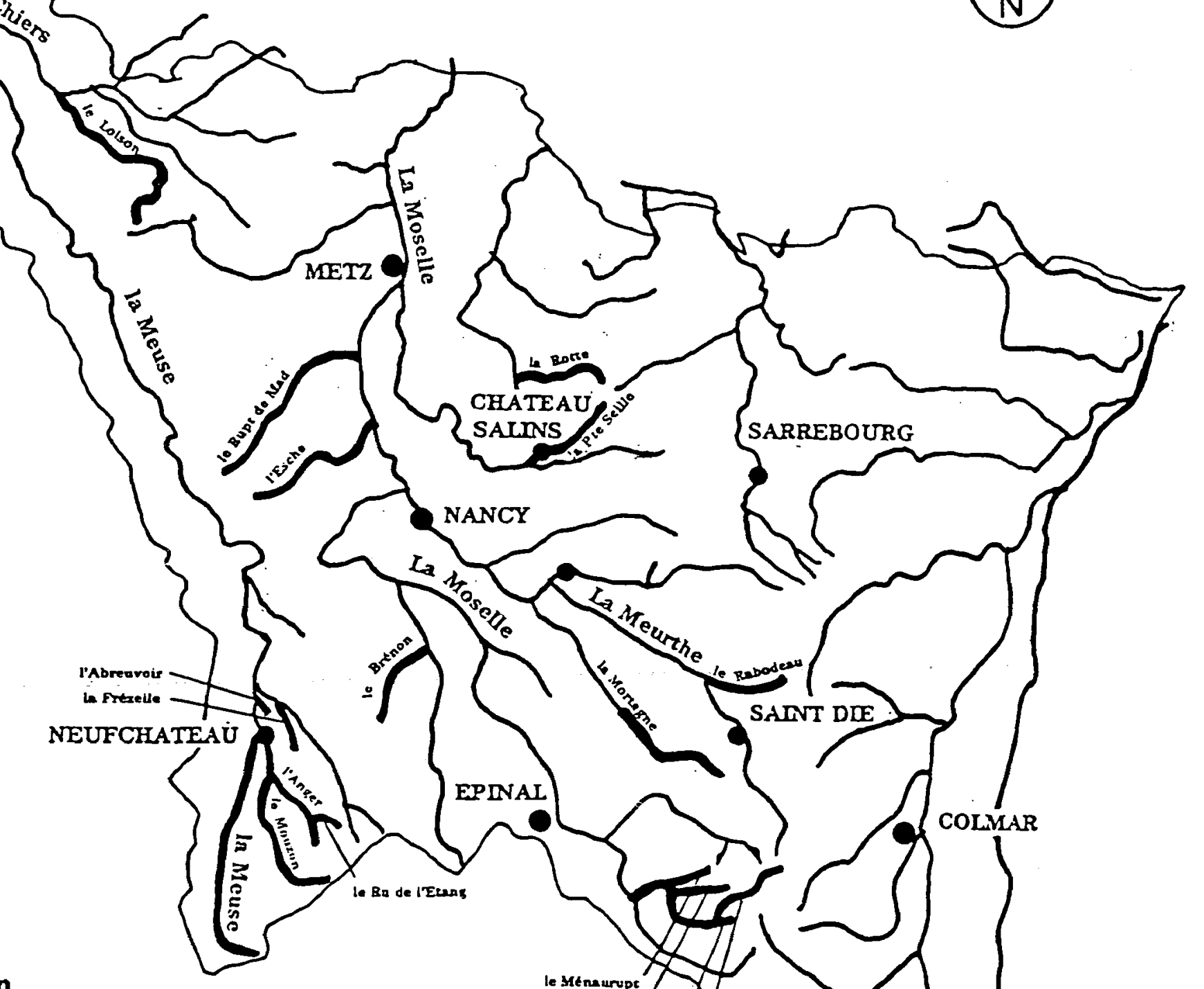

Fig. 1. Situation géographique des cours d'eau lorrains prospectés en 1990-1991.

Fig. 1. Location of the rivers studied in 1990-1991. 
Deux facteurs sont déterminants dans le choix des stations retenues qui, à l'échelle de ce travail, doivent rester homogènes : l'hydrodynamique locale (caractère lotique ou lentique), ceci tout particulièrement pour les grands cours d'eau, et l'éclairement, surtout pour les petits cours d'eau.

\subsection{Les descripteurs abiotiques du cours d'eau}

\subsubsection{Les facteurs géologiques}

Ils comprennent le socle géologique et les formations superficielles. Ils sont susceptibles d'influer sur le fonctionnement hydrologique quantitatif (pertes sur les substrats calcaires karstiques par exemple) mais aussi sur la composition chimique des eaux (Haslam \& Wolseley 1981, Haury 1985).

Dans cette étude cinq grands types de substrats géologiques ou classes de perméabilité (tableau 3) ont été individualisés : les granites (P33), les grès (P11), les marnes et les argiles (du Keuper, du Muschelkalk, du Rhétien et du Callovien) (P31), les calcaires (P12), et l'alternance marno-calcaire ou argilo-calcaire (P2).

Deux types de formations superficielles ont été distingués : perméables (S11) et peu perméables (S2).

\subsubsection{Les facteurs morphométriques}

Trois paramètres sont pris en compte :

- La hauteur d'eau moyenne est obtenue par une mesure sur le terrain (Mériaux \& Wattez 1980, HMSO 1987, Ramm 1988) ;

- La vitesse du courant est estimée par l'observateur selon une échelle de cinq classes : très rapide, rapide, moyenne, faible, très faible à nulle. C'est un paramètre important dans l'étude de l'eutrophisation (Haslam \& Wolseley 1981, Balocco-Castella 1988). Un courant élevé permet un renouvellement d'oxygène, d'où une amélioration de la capacité d'auto-épuration, une circulation des substances nutritives et une diminution de la capacité de réchauffement des eaux lors de fortes intensités lumineuses. D'autre part, il influe sur la répartition et l'abondance des espèces (Butcher 1933) ;

- La granulométrie du substrat correspond à une évaluation de l'observateur d'après l'échelle granulovisuelle de Cummins (in Malavoi 1988) : argiles, limons, sables, graviers, cailloux, blocs. La granulométrie est un des premiers facteurs de la répartition des algues filamenteuses et des macrophytes
(Barko \& Smart 1980, Wiegleb 1984), notamment des bryophytes (Haury 1985).

A ces trois facteurs fondamentaux s'ajoutent les largeurs en eau et entre berges ainsi que la hauteur des berges (mesurées sur le terrain), la pente moyenne (calculée à partir des cartes IGN) et l'ordre de drainage (calcul effectué d'après le réseau pérenne des cartes IGN $1 / 50000$, selon la règle suivante : la confluence de deux cours d'eau de même catégorie provoque la constitution d'un cours d'eau de rang supérieur, et un cours d'eau sans affluent est d'ordre 1).

\subsubsection{Les facteurs climatiques}

Le principal est l'éclairement reçu en surface car il est essentiel pour le développement de la végétation (Haury 1985, HMSO 1987, Balocco-Castella 1988, Bouxin 1991). Trois types d'évaluation de l'éclairement sont utilisés : une estimation visuelle par l'observateur ( 5 classes sont définies : très éclairé, éclairé, moyen, sombre, très sombre), une mesure à l'aide d'un luxmètre et une quantification par une méthode photographique.

Cette dernière utilisant des photographies hémisphériques a été mise au point par les forestiers (Becker 1971) et adaptée au milieu aquatique (Smith 1980, Levet 1989, Grasmück 1989). Elle permet de définir un pourcentage d'éclairement diffus et un pourcentage d'éclairement direct reçu sur la station. (Test méthodologique présenté in : Univ. de Metz, Laboratoire d'Ecologie et al., 1991).

Le deuxième facteur climatique pris en compte est la température de l'eau (mesure ponctuelle sur le terrain).

\subsubsection{Les facteurs chimiques}

Deux catégories de descripteurs chimiques ont été distinguées : les paramètres caractéristiques du niveau de trophie et ceux caractéristiques du niveau de minéralisation.

Les dosages et mesures ont été réalisés selon les normes françaises (AFNOR 1990).

Les paramètres caractéristiques du niveau de trophie sont :

- les orthophosphates et le phosphore total exprimés en $\mathrm{mgP} / 1$ (NF $\mathrm{T}$ 90-023);

- les nitrates (NF T 90-012), les nitrites (NF T 90-013) et l'azote ammoniacal (NF T 90-015), exprimés en $\mathrm{mgN} / 1$; 
Tableau 2. Présentation des principales caractéristiques des cours d'eau prospectés en 1990-1991.

Table 2. Main characteristics of the rivers studied in 1990-1991.

\begin{tabular}{|c|c|c|c|}
\hline \multirow{2}{*}{$\begin{array}{c}\text { Substrat géologique } \\
\text { dominant }\end{array}$} & \multicolumn{3}{|c|}{ Caractéristiques des cours d'eau } \\
\hline & Nom & Qualité 86-88 & Ordre de drainage \\
\hline \multirow{4}{*}{ GRANITES } & La Moselotte & $1 B-2-1 B$ & de 1 a 4 \\
\hline & Le Rouge Rupt & $1 A-1 B$ & de 1 a 3 \\
\hline & Le Bouchot & $1 A-1 B$ & de 1 a 3 \\
\hline & Le Ménaurupt & $1 \mathrm{~A}$ & de 1 a 2 \\
\hline \multirow[t]{2}{*}{ GRES VOSGIEN } & $\begin{array}{l}\text { La Mortagne } \\
\text { amont (conf. Arentèle) }\end{array}$ & $1 B 2-1 B$ & de 1 a 4 \\
\hline & Le Rabodeau & $1 \mathrm{~A}-1 \mathrm{~B}-2-1 \mathrm{~B}$ & de 1 a 4 \\
\hline $\begin{array}{c}\text { MARNES } \\
\text { Argiles et niveaux } \\
\text { dolomitiques du Keuper et } \\
\text { de la Lettenkohle } \\
\end{array}$ & $\begin{array}{l}\text { La Rotte } \\
\text { La Petite Seille } \\
\text { Ru de la Flotte } \\
\end{array}$ & $\begin{array}{c}2-1 B-3 \\
3-2 \\
1 B\end{array}$ & $\begin{array}{l}\text { de } 1 \text { à } 4 \\
\text { de } 1 \text { à } 3\end{array}$ \\
\hline \multirow{10}{*}{$\begin{array}{c}\text { Alternance } \\
\text { CALCAIRES/MARNES }\end{array}$} & $\begin{array}{l}\text { La Meuse } \\
\text { ( amont de Neufchâteau) }\end{array}$ & 2 & de 1 à 5 \\
\hline & L'Abreuvoir & & de 1 à 1 \\
\hline & Le Mouzon & $2-1 B$ & de 1 a 4 \\
\hline & L'Anger & $3-2-1 B-2-3-2-1 B$ & de 1 à 4 \\
\hline & Ru de l'Etang & M & de 1 à 4 \\
\hline & La Frézelle & $1 \mathrm{~A}$ & de 1 à 2 \\
\hline & Le Brénon & $1 B-M-3$ & de 1 à 3 \\
\hline & Ru du Moulin & & de 1 à 2 \\
\hline & Ru des Conrottes & & de 1 à 2 \\
\hline & L'Esche & $2-1 B-3$ & de 1 à 3 \\
\hline \multirow[t]{2}{*}{$\begin{array}{c}\text { ARGILES Amont } \\
\text { et } \\
\text { CALCAIRES Aval }\end{array}$} & Le Rupt de Mad & $2-1 B-2$ & de 1 à 4 \\
\hline & Le Loison & $2-1 B$ & de 1 à 4 \\
\hline
\end{tabular}


Tableau 3. Présentation des classes de perméabilité (d'après Zumstein et al. 1989).

Table 3. Introduction to the permeability classes (according to Zumstein et al. 1989).

\begin{tabular}{cccc}
\hline & PERMEABLES & Perméabilité dinterstice dominante : sables - grès & P11 \\
\cline { 2 - 4 } ROCHES & Perméabilité de fissures dominante : calcaires & P12 \\
\cline { 2 - 4 } & PEU PERMEABLES & Mélanges ou alternances de roches perméables \\
& & $\begin{array}{c}\text { (interstice ou fissures) et imperméables } \\
\text { Trois classes P21 P22 P23 regroupées }\end{array}$ & P2. \\
\cline { 2 - 4 } & IMPERMEABLES & Marnes et argiles & P31 \\
\cline { 2 - 4 } & & Roches compactes et compactées & P32 \\
\hline FORMATIONS & PERMEABLES & Granites & P33 \\
\cline { 2 - 4 } SUPERFICIELLES & PEU PERMEABLES & Sables - Graviers - Galets dominants & S11 \\
\hline
\end{tabular}

- la DCO (NF T 90-101) et la $\mathrm{DBO}_{5}(\mathrm{NF} \mathrm{T}$ 90-103), exprimées en $\mathrm{mg} \mathrm{d}^{\prime} \mathrm{O}_{2} / 1$;

- le $\mathrm{pH}$;

- l'oxygène dissous, exprimé en $\mathrm{mg} / \mathrm{l}$ et en pourcentage de saturation.

Les valeurs ponctuelles de $\mathrm{pH}$ et d'oxygène dissous (mesures effectuées in situ) n'auront qu'une valeur indicative en raison de la grande variabilité de ces paramètres au cours de la journée.

Les paramètres caractéristiques du degré de minéralisation sont :

- la conductivité exprimée en $\mu \mathrm{S} / \mathrm{cm}$ (mesure conductimétrique au laboratoire) ;

- la dureté (teneurs en calcium et magnésium, exprimées en $\mathrm{mg} / \mathrm{l}$ (NF T 90-005) ;

- le TA et le TAC exprimés en ${ }^{\circ} \mathrm{F}$ (NF $\mathrm{T}$ 90-036) ;

- les teneurs en sulfates exprimées en $\mathrm{mg} / \mathrm{l}(\mathrm{NF}$ T 90-040);

- les teneurs en sodium et en potassium exprimées en $\mathrm{mg} / \mathrm{l}$ (NF T 90-020) ;

- les teneurs en chlorures exprimées en mg/1 (NF T 90-014).

Tous les paramètres, excepté les cations, ont été dosés dans la journée de prélèvement.

\subsection{L'étude de la végétation aquatique}

Les stations d'étude ont une longueur minimale de $50 \mathrm{~m}$ afin de prendre en compte le maximum d'espèces présentes. Il est même recommandé d'augmenter cette longueur (jusqu'à 100,150 m) lorsque la richesse spécifique de la station est faible et à condition que celle-ci reste homogène.

Un inventaire qualitatif et quantitatif (à l'aide des pourcentages de recouvrement) est effectué. Il est constitué de la liste de toutes les macrophytes observées dans le lit en eau, soit les algues filamenteuses, les bryophytes et les végétaux supérieurs.

Du point de vue de la taxonomie, les bryophytes et les végétaux supérieurs seront identifiés jusqu'à l'espèce avec une attention toute particulière pour des groupes tels que les Callitriches et les Renoncules, les algues ne le seront que jusqu'au genre. Les principaux ouvrages de détermination utilisés ont été Augier (1966) pour les bryophytes, Bourrelly (1968, 1970, 1990) pour les algues et De Langhe et al. (1983) pour les végétaux supérieurs.

\subsection{L'exploitation des données}

L'exploitation donne lieu tout d'abord à une validation de la représentativité de notre échantillonnage et à une présentation de la liste floristique. 
Les résultats sont ensuite regroupés et étudiés au sein de chaque substrat géologique afin d'obtenir une première caractérisation physico-chimique et biologique et une description des successions végétales observées par type de cours d'eau.

Les relations entre espèces végétales et descripteurs sont ainsi mises en évidence puis discuitées.

\section{Résultats}

Les 17 cours d'eau et 4 .rus (Tableau 2) ont été prospectés durant les années 1990 et 1991. La matrice des résultats ainsi constituée contient 134 variables d'environnement et 119 stations d'étude.

\subsection{Représentativité de l'échantillonnage}

\subsubsection{Le choix des cours d'eau et des tronçons étudiés}

Les pourcentages d'occupation globale de chacune des classes de perméabilité ont été calculés à partir de données (Zumstein, comm. pers., d'après Gille 1983) concernant les bassins de la Moselle à l'amont de Sierck-les-Bains et de la Meuse à l'amont de Chooz ce qui inclut tous les cours d'eau prospectés. La comparaison des pourcentages d'occupation des classes de perméabilité et des pourcentages de stations prospectées montre que tous les substrats importants de Lorraine ont été échantillonnés.

Dans notre étude l'effort d'investigation s'est porté sur les granites (P33) qui occupent $8 \%$ du bassin Rhin-Meuse et qui constituent $19 \%$ de notre échantillon. Les grès (P11) et les marnes (P31), qui occupent respectivement 11 et $23 \%$ du bassin RhinMeuse, sont bien représentés, avec respectivement 20 et $43 \%$ de notre échantillon, par contre les alternances marnes-calcaires (P2) ont été quelque peu sous-échantillonnées : $17 \%$ d'occupation du bassin Rhin-Meuse et $5 \%$ de notre échantillon. Les calcaires (P12) occupent $22 \%$ du bassin Rhin-Meuse et représentent $14 \%$ de notre échantillon.

La majorité (74 \%) des stations prospectées a un ordre de drainage de $3(44 \%)$ ou $4(30 \%)$, les pourcentages de stations prospectées d'ordre 1 et d'ordre 2 sont faibles (respectivement $8 \%$ et $19 \%$ ).

La classe $\mathbf{P} 2$ est représentée exclusivement par des stations plutôt situées à l'aval des cours d'eau.

\subsubsection{Les végétaux rencontrés}

Soixante-dix taxons aquatiques ont été inventoriés : 2 genres d'algues bleues, 15 genres d'algues filamenteuses, 6 espèces de mousses, 1 genre de sphaignes, 2 espèces d'hépatiques et 44 espèces de plantes supérieures.

La richesse moyenne par station est de 8 taxons, pouvant aller jusqu'à 18 et, sans les populations algales, elle descend à une valeur móyenne de 6 .

Ce sont des algues du genre Vaucheria qui sont retrouvées le plus fréquemment (sur $45 \%$ des stations): Les autres taxons les plus récoltés (sur 30 à $36 \%$ des stations) sont constitués par des algues du genre Cladophora et des phanérogames : Phalaris arundinacea L., Sparganium erectum L., S. emersum Rehm. et Iris pseudacorus L..

Ensuite une espèce de mousses, neuf espèces de phanérogames et deux genres d'algues sont présents sur 20 à $30 \%$ des stations; dans l'ordre décroissant de présence Fontinalis antipyretica Hedw., Lemna minor L., Glyceria fluitans (L.) R. Brown, Ulothrix sp, Butomus umbellatus L., Nuphar lutea (L.) Smith, Solanum dulcamara L., Glyceria maxima (Harmt.) Holmberg, Callitriche hamulata Kütz. ex. Koch, Callitriche platycarpa Kütz., Beruila erecta (Huds.) Coville et Enteromorpha sp.

Sur 8 à $17 \%$ des stations, on retrouve les espèces ou taxons suivants (toujours dans l'ordre décroissant de présence); Veronica anagallisaquatica L., Scapania undulata (L.) Dum., algues bleues, Potamogeton crispus L., Spirogyra sp, Potamogeton pectinatus L., Ranunculus fluitans Lam., Zannichellia palustris L., Myriophyllum spicatum L., Mentha aquatica L., Brachythecium rivulare B.e., Veronica beccabunga L., Amblystegium riparium Hedw. (synonyme Leptodictyum riparium (Hedw.) Warnst., Nasturtium officinale R. Brown.

Les espèces ou taxons restants n'ont pas été souvent rencontrés du fait de leur rareté ou de substrats favorables peu échantillonnés : Oscillatoria $s p$, Chiloscyphus polyanthus (L.) Corda, Hygrohypnum dilatatum (Wils.) Loeske, Octodiceras fontanum (La Pyl.) Lindb., Callitriche obtusanguia Le Gall, $C$. stagnalis Scop., Elodea canadensis Michaux et $E$. nuttallii (Planch.) St John, Cardamine amara L., Ranunculus peltatus Schrank et $R$. penicillatus (Dum.) Bab., Sagittaria sagittifolia L., Potamogeton natans L., $P$. perfoliatus L., $P$. lucens L., $P$. berchtoldii Fieb., Oenanthe aquatica (L.) Poiret. 


\subsection{Caractérisation physico-chimique et biologique des classes de perméabilité et successions végétales des cours d'eau étudiés}

Chacune des principales classes de perméabilité étudiées peut être caractérisée par un certain nombre de variables d'environnement et de biologie.

Les variables caractéristiques sont représentées par leur valeur moyenne pour cette classe. Elles sont rangées par ordre d'importance selon un critère statistique : la valeur test ; plus celle-ci est élevée et plus la variable est caractéristique. La caractérisation s'obtient par un test du Chi 2 comparant le profil de la classe pour une variable donnée au profil global de cette variable pour l'ènsemblè de là matrice en utilisant le nombre d'écarts types.

On attribue un signe à la valeur test. Si le terme est positif, la classe est caractérisée par les valeurs fortes de la variable et inversement si le terme est négatif, ce seront les valeurs faibles de la variable qui caractériseront la classe (la valeur test est significative hors de l'intervalle allant de -2 à +2 ).

Ce tri est réalisé par la procédure de description DEMOD du logiciel statistique SPAD.N 2.O (CISIA 1991).

\subsubsection{Les cours d'eau sur grès P11}

- Caractérisation statistique de la classe des grès (24 stations)

Une première exploitation des résultats (Léglize \& Grasmück 1992) a montré que la classe des grès devrait être divisée en deux : les stations P11 des cours d'eau s'écoulant exclusivement sur grès et les stations de cours d'eau sur grès en alternance avec des couches argilo-calcaires ou marno-calcairès, que l'on nommera désormais P13, par commodité, mais qu'il n'y a pas lieu de considérer comme une nouvelle classe de perméabilité au sens strict.

Cette opposition est très marquée pour les valeurs de minéralisation et de trophie de l'eau. Entre les stations sur P11 et les stations sur P13, la conductivité moyenne augmente de 17 fois sa valeur (de 68 à $1200 \mu \mathrm{S} / \mathrm{cm})$.

La classe des grès purs P11 (14 stations) est caractérisée (Tableau 4) :

par des variables morphométriques : substrat sableux, eau claire, environnement forestier et éclairement diffus faible $(27 \%)$;
- par des variables de végétation : Batrachospermum sp., Chiloscyphus polyanthus (L.) Corda, des sphaignes, Hygrohypnum dilatatum (Wils.) Loeske et Ranunculus peltatus Schrank (espèces dès eaux peu minéralisées). Un pourcentage de fơnd nu important ( $82 \%$ ) est également représentatif de ces stations sur grès purs.

- par des variables chimiques de minéralisation (TAC de $1,4^{\circ} \mathrm{F}, \mathrm{Ca}++4 \mathrm{mg} / 1$, conductivité $68 \mu \mathrm{S} / \mathrm{cm}$ ) caractéristiques de cette classe par leurs faibles teneurs (valeurs tests négatives).

La classe $\mathrm{P} 13$ des grès succédant à des alternances est caractérisée (Tableau 4) par des variables de végétation : Vaucheria sp (plutôt ubiquiste mais rare dans les eaux peu minéralisées) et trois espèces de phanérogames Sparganium erectum L., Potamogeton natans L., Butomus umbellatus L..

Cette classe est caractérisée également par deux variables de chimie (TAC $28,5^{\circ} \mathrm{F}$ et $\mathrm{K}+7,2 \mathrm{mg} / \mathrm{l}$ ).

Les deux classes n'ont aucune variable significative commune. L'opposition entre «P11 " et «P13 » est donc confortée. Cette analyse souligne l'importance de l'influence des écoulements amont car ces stations sur grès (P13) n'offrent plus du tout les caractéristiques attendues des grès (P11). Ces milieux de perméabilité P11 sont particulièrement sensibles à tout apport polluant et ainsi très fragiles.

- Succession végétale longitudinale des cours d'eau s'écoulant exclusivement sur grès purs P11

Le gradient type de végétation aquatique est illustré par la Mortagne (amont de Rambervillers). Il résulte d'une situation quasi-naturelle. A l'amont, on y observe des bryophytes et, en particulier, Chiloscyphus.polyanthus.(L.) Corda et Scapania undulata (L.). Dum.. Cette association est souvent rencontrée dans les cours d'eau du Massif Armoricain (Haury \& Muller, 1991). D'autres bryophytes sont également présentes: Hygrohypnum dilatatum (Wils.) Loeske, Hypnum cupressiforme Hedw. et Fontinalis antipyretica Hedw.. Puis à l'aval, Ranunculus peltatus Schrank et des callitriches sont rencontrés. Callitriche hamulata Kütz. ex. Koch est d'ailleurs présent dans toutes les stations.

Potamogeton polygonifolius Pourr. a été récolté sur une station amont, c'est un bon indicateur de milieu peu minéralisé et oligotrophe (Muller 1990b) et sa disparition est synonyme d'un enrichissement en nutriments. 
Tableau 4. Exemple des variables physiques, chimiques et biologiques caractéristiques des classes P11 «grès purs » et P13 « grès d'alternances " issues de la méthode statistique de description.

Table 4. Physical, chemical and biological variables of the P11 and P13 classes.

\begin{tabular}{|c|c|c|c|}
\hline \multirow{2}{*}{$\begin{array}{l}\text { Variables caractéristiques } \\
\text { P11 : Grès "purs" } \\
\end{array}$} & \multicolumn{2}{|c|}{ Moyennes } & \multirow{2}{*}{$\begin{array}{c}\text { Valeur } \\
\text { test }\end{array}$} \\
\hline & Classe & Générale & \\
\hline Batrachospermum sp & 0,29 & 0,03 & 5,55 \\
\hline Cardamine amara & 0,29 & 0,04 & 4,82 \\
\hline Hygrohypnum dilatatum & 3,14 & 0,46 & 4,55 \\
\hline Fond nu (\%) & 82 & 46 & 4,44 \\
\hline Végétation totale (\%) & 17,5 & 54 & $-4,44$ \\
\hline Ranunculus peltatus & 2,21 & 0,29 & 3,96 \\
\hline Chiloscyphus polyanthus & 0,86 & 0,11 & 3,83 \\
\hline Sphagnum sp & 0,43 & 0,06 & $3 ; 1$ \\
\hline Pourcentage algues filamenteuses & 2,5 & 30 & $-3,48$ \\
\hline Pourcentage bryophytes & 9 & 4 & 2,82 \\
\hline Scapania undulata & 3,5 & 1 & 2,8 \\
\hline Pente berge Rive Gauche (\%) & 90 & 70,5 & 3,84 \\
\hline Pente berge Rive Droite (\%) & 90 & 70,5 & 3,79 \\
\hline Eclairement direct (\%) & 19,5 & 49,5 & $-3,5$ \\
\hline Eclairement diffus (\%) & 27 & 55 & $-3,61$ \\
\hline Conductivité en $\mu \mathrm{S} / \mathrm{cm}$ & 68 & 793 & $-3,85$ \\
\hline Calcium en $\mathrm{mg} / \mathrm{l}$ & 4 & 94 & $-4,74$ \\
\hline TAC en ${ }^{\circ} \mathrm{F}$ & 10,4 & 17,3 & $-5,22$ \\
\hline pH & 6,3 & 7,5 & $-5,75$ \\
\hline \multicolumn{4}{|l|}{ P13 : Grès en alternance } \\
\hline Sparganium erectum & 11,5 & 2 & 3,94 \\
\hline Vaucheria sp & 39 & 13 & 3,86 \\
\hline Potamogeton natans & 0,5 & 0,05 & 3,18 \\
\hline Butomus umbellatus & 3,7 & 1 & 2,5 \\
\hline TAC en ${ }^{\circ} \mathrm{F}$ & 28,5 & 17,3 & 3,05 \\
\hline Potassium en $\mathrm{mg} / \mathrm{l}$ & 7,2 & 4,5 & 2,39 \\
\hline
\end{tabular}

3.2.2. Les stations de cours d'eau s'écoulant sur calcaires P12 (16 stations)

La classe des stations sur calcaires est caractérisée par une variable morphométrique (tracé de lit courbe), et surtout par des variables de végétation, en particulier une richesse taxonomique importante (12 taxons), un genre d'algues bleues Oscillatoria sp, une mousse Fontinalis antipyretica Hedw. et plusieurs espèces de phanérogames Ranunculus fluitans Lam., Oenanthe fluviatilis (Bab.) Colem., Callitriche obtusangula Le Gall, Acorus calamus L. et Elodea canadensis Michaux.
Les stations sur calcaires sont toutes situées à l'aval des cours d'eau (excepté les deux stations de l'Abreuvoir, qui reste un cas un peu particulier). Elles sont caractérisées par l'apparition de Ranunculus fluitans Lam., mais aussi Oenanthe fluviatilis (Bab.) Colem. et Sagittaria sagittifolia L.

En outre, dans ces stations, sont présents Amblystegium riparium $\mathrm{Hedw}$. et Fontinalis antipyretica Hedw..

Cladophora sp et Vaucheria sp sont les deux principaux genres d'algues inventoriés. 
3.2.3. Les stations de cours d'eau s'écoulant sur un substrat peu perméable $P 2$ résultant d'une alternance de roches perméables et imperméables (6 stations)

L'effectif de cette classe est faible et ne permet donc pas une étude approfondie. La variable caractéristique de cette classe est une mousse Amblystegium riparium Hedw., puis viennent des variables chimiques $\left(\mathrm{K}^{+} 9 \mathrm{mg} / \mathrm{l}, \mathrm{DBO}_{5} 6 \mathrm{mg} / \mathrm{l}\right.$ et $\mathrm{DCO}$ $20 \mathrm{mg} / \mathrm{l})$.

\subsubsection{Les cours d'eau sur marnes P31}

- Caractérisation statistique de la classe des marnes (51 stations)

La classe des stations sur marnes est caractérisée par des variables chimiques et notamment une minéralisation forte $(\mathrm{Ca}++142 \mathrm{mg} / \mathrm{l}$, conductivité $1240 \mu \mathrm{S} / \mathrm{cm}, \mathrm{Mg}++44 \mathrm{mg} / \mathrm{l}, \mathrm{TAC} 23,7^{\circ} \mathrm{F}$ ), par des variables morphométriques (substrat limoneux, berges en terre, parcelles avoisinantes pâturées) et par une végétation totale importante (en moyenne $70 \%$ de recouvrement), un genre d'algues Cladophora sp et une espèce de phanérogames : Potamogeton pectinatus L. (espèce caractéristique des eaux très minéralisées). Les stations sur marnes ont un indice d'éclairement direct fort, synonyme d'ensoleillement (une moyenne de $63,5 \%$ contre seulement $49 \%$ pour la totalité des relevés).

- Successions végétales longitudinales

- Des cours d'eau s'écoulant exclusivement sur marnes (marnes du Keuper, en particulier)

Ils se caractérisent par la quasi-absence des bryophytes ; toutefois une mousse apparaît parfois en faible quantité, Fontinalis antipyretica Hedw. Le substrat fin argilo-marneux n'est en effet pas favorable à la colonisation par des mousses.

Ces cours d'eau marneux situés en zone agricole ne présentent pas de gradient de végétation mais plutôt une combinaison type : des algues vertes très abondantes (Cladophora sp, Enteromorpha sp, Vaucheria sp, etc...) et le potamot pectiné (Potamogeton pectinatus L.):

- Des cours d'eau s'écoulant sur des alternances (de type calcaires-grès-marnes)

Les stations de perméabilité P31 issues de cours d'eau résultant d'alternances présentent une végétation aquatique plus diversifée que les stations (P31) des cours d'eau s'écoulant exclusivement sur marnes. L'effet « marnes » est légèrement estompé par des influences amont (excepté pour l'amont de l'Anger qui se trouve être dans les marnes irisées du même type que celles du Keuper).

On retrouve des algues filamenteuses en fortes proportions (Enteromorpha sp, Cladophora sp, Vaucheria sp, Spirogyra sp) et des espèces caractéristiques des milieux eutrophes (De Langhe et al. 1983, Muller 1990a) : Potamogeton pectinatus L., $P$. crispus L., $P$. perfoliatus L., Sparganium emersum Rehm., S. erectum L., Elodea canadensis Michaux, Myriophyllum spicatum L., Zannichellia palustris L., Berula erecta (Huds.) Coville, Nuphar lutea (L.) Smith, Callitriche obtusangula Le Gall et C. platycarpa Kütz.

Les successions naturelles, sur ces cours d'eau résultant d'alternances, ne sont pas faciles à déceler. Les algues filamenteuses dominent souvent à l'amont tandis qu'à l'aval les recouvrements et la richesse en phanérogames augmentent.

C'est dans ces cours d'eau s'écoulant sur des alternances que les stations sur grès $P 11$ deviennent des stations de type P13, c'est-à-dire qu'elles sont beaucoup plus proches au niveau de leur végétation et de leurs caractéristiques physico-chimiques des stations sur marnes $\mathrm{P} 31$ que des stations sur grès purs.

\subsubsection{Les cours d'eau sur granites $P 33$}

- Caractérisation statistique de la classe des granites (22 stations)

\section{La classe des granites est caractérisée par}

- des variables morphométriques : une pente forte (une moyenne de 33 pour mille), des berges enrochées, des formations superficielles de types limons S2, une vitesse de courant très rapide, un substrat fait de blocs, une eau claire

- des variables de végétation : des algues bleues, un genre d'algues rouges Lemanea sp, des mousses et hépatiques Brachythecium rivulare B. e., Scapania undulata (L.) Dum., et des phanérogames Ranunculus cf penicillatus (Dum) Bab. et Callitriche hamulata Kütz ex. Koch (des espèces d'eaux peu minéralisées), l'élément majeur étant l'importance du fond nu (un recouvrement de $87 \%$ ), tout comme la classe des grès " purs » $\mathrm{P} 11$.

- Succession longitudinale des cours d'eau sur granites

Les taxons inventoriés à l'amont sont des algues bleues, des hépatiques notamment Scapania 
undulata (L.) Dum. et des mousses Brachythecium rivulare B. e., B. plumosum (Sw.) B. e., Amblystegium riparium Hedw. et Fontinalis antipyretica Hedw..

A l'aval on rencontre des algues vertes ainsi que Callitriche hamulata Kütz. ex. Hoch, C. platycarpa Kütz. et Ranunculus peltatus Schrank.

Ranunculus penicillatus (Dum.) a également été récolté (sur la Moselotte en particulier). Cette espèce est plus fréquente en milieu océanique (Haury \& Muller 1991) et, comme sa détermination reste délicate, nous préférons la nommer $R$. cf penicillatus.

\subsection{Bilan}

L'hétérogénéité des types géologiques de Lorraine réapparaît au niveau de cet échantillonnage, de par les résultats obtenus aussi bien au niveau :

- de la morphométrie : des cours d'eau de montagne aux fortes pentes (massif vosgien : la Moselotte, le Bouchot, etc...) aux cours d'eau de plaine aux pentes très faibles (la région du Bassigny : la Meuse amont, le Mouzon, l'Anger, etc...) ;

- de la chimie : des cours d'eau très peu minéralisés sur granites et grès purs aux cours d'eau très minéralisés sur marnes ;

- de la végétation : des hépatiques (Scapania undulata (L.) Dum. et Chiloscyphus polyanthus (L.) Corda à des espèces telles que Potamogeton pectinatus L..

Cette première exploitation des résultats aboutit à l'élaboration d'une présentation synthétique des classes de perméabilité et de la distribution longitudinale des cortèges floristiques (Tableau 5). Elle valide le choix de cette classification comme base d'une typologie.

\section{Discussion}

Les inventaires de végétation effectués sont très hétérogènes en quantité et en qualité à l'échelle de la Lorraine.

La notion de typologie des cours d'eau par les macrophytes va être abordée en distinguant deux grandes catégories : les cours d'eau avec une végétation peu abondante (recouvrement de végétation totale $<50 \%$ ) qui sont en fait les cours d'eau sur substrats siliceux et les autres dotés d'une végétation abondante (recouvrement $>50 \%$ ) qui sont les cours d'eau sur substrats argileux.
Une végétation typique ainsi que des gradients longitudinaux ont été mis en évidence pour des cours d'eau situés dans des conditions extrêmes. Les relations entre espèces végétales et descripteurs sont maintenant commentées.

\subsection{Les cours d'eau sur substrats siliceux}

Ce sont principalement les cours d'eau situés sur les granites $(\mathrm{P} 33)$ et les grès $(\mathrm{P} 11)$.

- Les cours d'eau sur granites

Toutes les espèces présentes sont caractéristiques des eaux très faiblement minéralisées et oligotrophes (Augier 1966, De Langhe et al. 1983, Muller 1990a, Haury \& Muller 1991).

Scapania undulata (L.) Dum. est typique des petites rivières à substrat rocheux avec une eau claire et un courant rapide (Jeffries \& Mills 1990) et caractérise des milieux oligotrophes (Haury 1985). Les bryophytes peuvent supporter de forts ombrages (Jeffries \& Mills 1990) ce qui est souvent le cas des stations amont sous forêt d'épicéas. Elles sont également capables de vivre dans des eaux acides (cas $\mathrm{du}$ Xoulces amont ( $\mathrm{pH}=5,7)$ notamment).

L'apparition des phanérogames à l'aval semble liée à l'augmentation des matières nutritives dans les eaux.

Les teneurs en phosphates augmentent de l'amont vers l'aval de 0,02 à $0,23 \mathrm{mgP} / 1$ pour la Moselotte, ainsi que les teneurs en nitrates de 0,1 à $0,5 \mathrm{mgN} / 1$, alors que les teneurs en ammoniaque restent stables $0,04 \mathrm{mgN} / \mathrm{l}$. Pour le Xoulces c'est essentiellement l'accroissement des concentrations en azote $\left(\mathrm{NH}_{4}{ }^{+}\right.$ de 0,01 à $0,13 \mathrm{mgN} / 1$ et $\mathrm{NO}_{3}-$ de 0,1 à $0,3 \mathrm{mgN} / \mathrm{l}$ ) qui caractérise l'enrichissement du milieu.

Cet enrichissement peut provenir d'une sédimentation due aux changements notables de pente et de largeur mais également aux apports des activités anthropiques de la vallée (villes, industries).

- Les cours d'eau sur grès purs

Toutes les espèces rencontrées sont familières des cours d'eau peu minéralisés à tendance oligotrophe et mésotrophe (Augier 1966, De Langhe et al. 1983, Muller 1990a).

Callitriche hamulata Kütz ex. Koch a cependant été récolté sur d'autres types de stations notamment sur l'Esche et ne semble donc pas être inféodé exclusivement aux eaux très peu minéralisées, ce qui est confirmé par Haury (1990). 

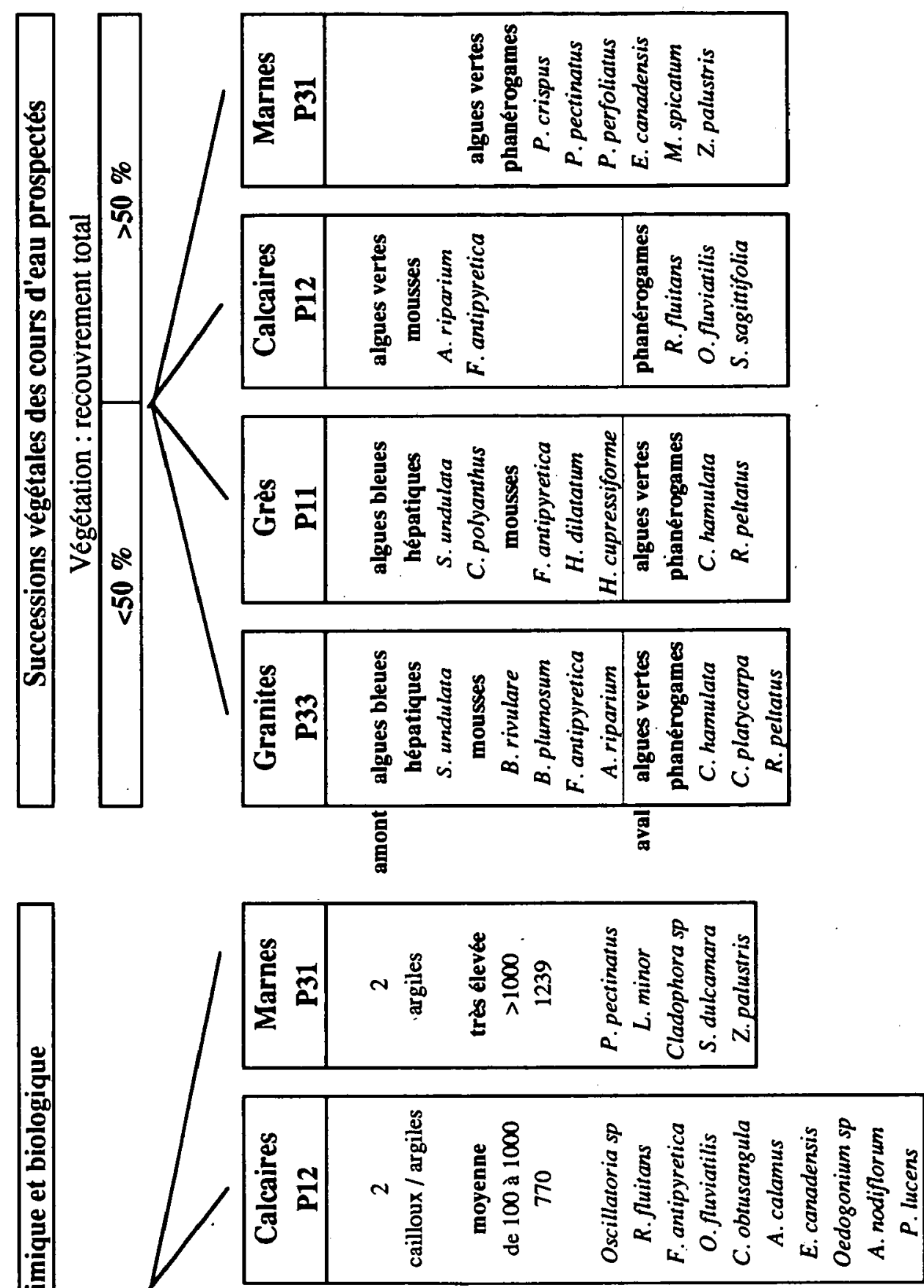

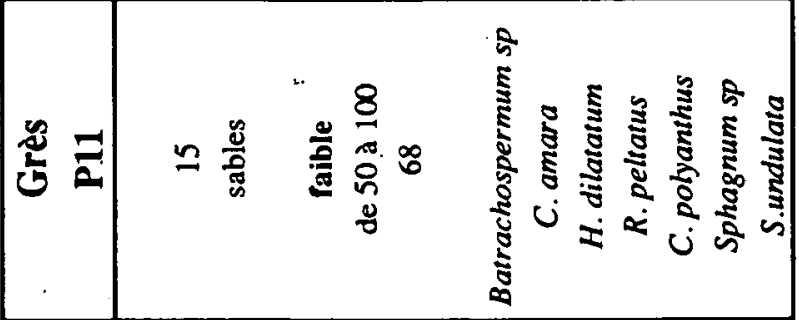

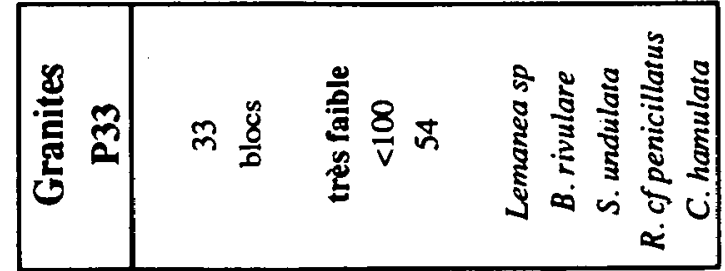

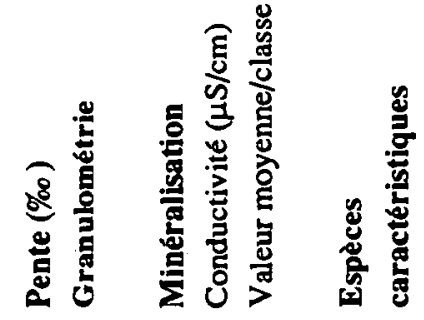


- Bilan des cours d'eau sur substrats siliceux

Les cours d'eau sur substrats siliceux présentent une végétation surtout constituée par des hépatiques et des mousses.

On retrouve avec ce bilan les relations citées par les auteurs entre la végétation aquatique et les caractéristiques de minéralisation et de trophie des eaux.

Les successions végétales étudiées (Tableau 5) sont intéressantes car elles traduisent l'évolution longitudinale du cours d'eau par des disparitions d'espèces (Potamogeton polygonifolius Pourr. et Scapania undulata (L.) Dum.) ou par l'apparition de taxons nouveaux : les algues vertes et les renoncules.

\subsection{Les cours d'eau sur substrats argileux}

Ce sont tous les autres cours d'eau prospectés sur les marnes et les alternances calcaires-marnes et argiles-calcaires.

\section{- Les cours d'eau sur marnes du Keuper}

L'espèce la plus fréquemment rencontrée dans ces cours d'eau est le potamot pectiné, Potamogeton pectinatus L., susceptible de supporter les plus fortes pollutions (Diederich 1983), et caractéristique des eaux eutrophes. Il peut d'ailleurs, sur certaines stations, présenter des populations monospécifiques.

De plus, les cladophores, également familières de ces milieux, sont connues dans des eaux chargées en matières azotées (Butcher 1933) et également bien chargées en phosphates (Ten Cate et al. 1991).

A la vue des gammes de minéralisation et de trophie naturelles très élevées de ces milieux sur marnes du Keuper (une conductivité qui avoisine les $2000 \mu \mathrm{S} / \mathrm{cm}$ et des teneurs en $\mathrm{PO}_{4}^{--}$variant entre 0,4 et $1,7 \mathrm{mgP} / \mathrm{l}$ ) et, par comparaison avec des milieux beaucoup moins minéralisés tels que ceux étudiés auparavant sur substrats siliceux, ces systèmes paraissent peu sensibles à divers apports polluants. En réalité, le seuil de l'hypereutrophisation peut être rapidement atteint. Ainsi, ces milieux peuvent être plus facilement sujets à des proliférations.

\section{- Les cours d'eau sur " alternances"}

Ces cours d'eau sont des rivières de plaine avec des courants faibles et qui sont, pour la plupart, recalibrés. Ils ont également souvent à supporter des rejets polluants : villes, exploitations agricoles, industries. De ce fait, la succession végétale résulte des inter-relations complexes entre tous ces paramètres.
- Bilan des cours d'eau sur substrats argileux

Sur ces cours d'eau s'écoulant sur substrats argileux, la végétation aquatique est typique de milieux bien minéralisés avec un niveau trophique important. Ici l'évolution longitudinale se traduit par l'augmentation de la biomasse de la végétation avec une tendance à la suprématie d'une espèce, par exemple le potamot pectiné (Tableau 5).

\section{Conclusion}

Cette étude de la végétation aquatique en Lorraine a permis de mettre en évidence plusieurs catégories de cours d'eau en fonction de leur végétation compagne.

L'utilisation de la géologie et plus précisément de la perméabilité de chaque station d'étude a permis de bien resituer les grands types géologiques dans lesquels la végétation se place. Il faut cependant se montrer prudent quant à l'utilisation de ces classes de perméabilité lorsque l'on est en présence d'alternances de substrats géologiques, notamment pour des milieux originellement fragiles comme les grès purs (P11) avec le cas des P13 « d'alternances »; ceci implique de s'intéresser aussi à la perméabilité amont du cours d'eau.

La pertinence de ce premier critère hiérarchique de classification des cours d'eau (classes de perméabilité) est confirmée par les résultats de cette étude. En effet, à chaque classe de perméabilité est associé un ensemble de variables d'environnement significatives, ce qui s'avère pertinent pour des études ultérieures et notamment des suivis de stations.

La végétation aquatique est un outil performant de caractérisation des milieux car elle intègre, par sa présence ou son abondance, des variations aussi bien morphométriques (éclairement, substrat ou courant) que chimiques. Ce type de caractérisation est d'autant plus intéressant que les macrophytes sont facilement observables et cartographiables dans les cours d'eau. Certaines espèces peuvent être considérées comme bioindicatrices, particulièrement dans des conditions extrêmes de minéralisation et de trophie. Potamogeton pectinatus L. et Myriophyllum spicatum L. par exemple.

L'évolution longitudinale de la végétation aquatique des cours d'eau de Lorraine, mise en évidence ici, sera précisée par une étude sur plusieurs périodes pour pouvoir caractériser les variations saisonnières de ces peuplements. 
Cette étude permettra en outre une mise en évidence des espèces indicatrices par la méthode des profils écologiques. Elle est d'ores et déjà utilisée pour la mise en œuvre d'indices macrophytes et des analyses multidimensionnelles permettront d'affiner les typologies présentées et contribueront à la définition de phytocénoses de référence.

\section{Remerciements}

Nous remercions MM. J.P. Décloux (GEREEA), P. Paris (AREA) qui ont réalisé les découpages abiotiques des cours d'eau prospectés et M. P. Rousselle (Univ. de Metz) qui a réalisé les dosages en spectrométrie d'absorption atomique.

Cette étude a été réalisée dans le cadre d'un travail financé par l'Agence de l'Eau Rhin-Meuse.

\section{Travaux cités}

AFNOR 1990. - Recueil de normes françaises. Eaux - Méthodes d'essai. 4ème éd. Paris. AFNOR. 736 p.

Agence de l'Eau Rhin-Meuse 1982. - Carte hydrogéologique du Bassin Rhin-Meuse.

Agence de l'Eau Rhin-Meuse 1989. - Carte de qualité des eaux superficielles du Bassin Rhin-Meuse. Actualisation 1986-1988.

Agence de l'Eau Seine Normandie 1989. - Eutrophisation. Réunion du 24 février 1989. $23 \mathrm{p}$.

Augier J. 1966. - Flore des Bryophytes. Paris. P. Lechevallier, $702 \mathrm{p}$.

Balocco-Castella C. 1988. - Les macrophytes aquatiques des milieux abandonnés par le Haut Rhône et l'Ain : diagnostic phyto-écologique sur l'évolution et le fonctionnement de ces écosystèmes. Th. Doct. : Univ. de Lyon I : $150 \mathrm{p}+$ annexes.

Barko J.W. \& Smart R.M. 1980. - Mobilization of sediment phosphorus by submersed freshwater macrophytes. Freshw. Biol., 10, 229-238.

Becker M. 1971. - Une technique nouvelle d'utilisation des photographies hémisphériques pour la mesure du climat lumineux en forêt. Ann. Sci. For., 28 (4), 425-442.

Bourrelly P. 1968-1970-1990. - Les algues d'eau douce. Paris, Boubée, 3 tomes, 438 p., 512 p. et 572 p.

Bouxin G. 1991. - La végétation aquatique et du bord de l'eau dans le bassin versant du Bocq (Condroz, Belgique). Rev. Sci. Eau, 4, 185-210.

Butcher R.W. 1933. - Studies on the ecology of rivers. I. On the distribution of macrophytic vegetation in the rivers of Britain. J. Ecol. 21 : 58-91.

CISIA 1991. (Centre International de Statistique et d'Informatique Appliquées), SPAD N. 2.0 intégré. St Mandé (France).

De Langhe J.E., Delsovalle L., Duvigneaud J., Lambinon J. \& Vanden Berghen C. 1983. - Nouvelle Flore de la Belgique, du Grand Duché de Luxembourg, du Nord de la France et des régions voisines (Ptéridophytes et Spermaphytes). $3^{\mathrm{e}}$ éd. Belgique, Meise. Ed. Patrim. Jard. bot. natl, 1015 p.

Diederich P. 1983. - Le genre Potamogeton L. s.l. au Grand Duché de Luxembourg et dans les régions voisines. Dumortiera, $27: 26-36$.

Gille E. 1983. - Le fichier physiographique des bassins de la Meuse et de la Moselle et son programme d'appel. CIG, AFBRM, ORSTOM.
Grasmück N. 1989. - Etude des relations entre végétaux fixés et caractéristiques du milieu : approche bibliographique et méthodologique de la quantification de la biomasse et de l'éclairement. Mém. D.E.A. : Sci. Agron. : ENSAIA, Nancy, 1-71 + annexes.

Haslam S.M. \& Wolseley P.A. 1981. - River vegetation, its identification, assessment and management. Cambridge, Cambridge Univ. Press, $154 \mathrm{p}$.

Haury J. 1985. - Etude écologique des macrophytes du Scorff (Bretagne-Sud). Th. Doct. : Ecologie. Rennes I, 240 p.

Haury J. 1990. - Macrophytes du Trieux (Bretagne-Nord) : III - Relations macrophytes - qualité des eaux. Bull. Soc. Nat. Ouest de la France, nouvelle série, tome 12 (4), p. 141-154.

Haury J. \& Muller S. 1991. - Variations écologiques et chorologiques de la végétation macrophytique des rivières acides du Massif armoricain et des Vosges du.Nord (France). Rev. Sci. Eau, 4, 463-482.

HMSO (Her Majesty's Stationery Office, London), Haslam S.M., Holmes N.T.H. \& Harding J.P.C. 1987:-Methods for the use of aquatic macrophytes for assessing water quality 1985-86 : methods for the examination of waters and associated materials. London, HMSO, $176 \mathrm{p}$.

Jeffries M. \& Mills D. 1990. - Freshwater Ecology - Principles and applications. London, Belhaven Press, 285 p.

Léglize L. \& Grasmück N. 1992. - Etụde des végétaux aquatiques des cours d'eau lorrains. Rapp. de contrat Univ. de Metz (Lab. d'Ecologie)/Agence de l'Eau Rhin-Meuse, 2 vol. : $42 \mathrm{p}$ + bibliogr. annexes.

Léglize L., Peltre M.C., Décloux J.P., Duval T., Paris P. \& Zumstein J.F. 1990. - Caractérisation des milieux aquatiques d'eaux courantes et végétation fixée. $14^{e}$ Conf. Int. COLUMA, Versailles 23-24 Janvier : 237-245.

Levet D. 1989. - Une méthode de mesure de l'éclairement audessus des rivières. Groupe Végétaux aquatiques de l'A.N.P.P. Réunion du 9 mars $1989: 6$ p.

Malavoi J.R. 1988. - Protocole de description des composantes morphodynamiques d'un cours d'eau à fond caillouteux. CEMAGREF, Lyon, $26 \mathrm{p}$.

Mériaux J.L. \& Wattez J.R. 1980. — Les végétations aquatiques et subaquatiques des eaux stagnantes et courantes du Nord de la France. Relations avec les composantes chimiques des eaux. In : La pollution des eaux continentales. Paris, Gauthiers-Villars, $2^{e}$ éd. (Pesson P. \& al.), pp. 224-242.

Muller S. 1990a. - Végétaux aquatiques dans les rivières du Bassin Rhin-Meuse. Synthèse bibliographique. Rapport Agence de l'Eau Rhin-Meuse. 45 p.

Muller S. 1990b. - Une séquence de groupements végétaux bioindicateurs d'eutrophisation croissante des cours d'eau faiblement minéralisés des Basses Vosges gréseuses du Nord. C.R. Acad. Sci. Paris, t. 310, Série III, p. 509-514.

Ramm A.E. 1988. - The community degradation index : a new method for assessing the deterioration of aquatic habitats. Water Res., Vol. 22, n 3, p. 293-301.

Smith B.D. 1980. - The effects of afforestation on the trout of a small stream in Southern Scotland. Fish. Manage, 11 (2), 39-58.

Strahler A.N. 1964. - Quantitative geomorphology of drainage basins and channel networks. Section 4. II. Part. In : Handbook of applied hydrology - A compendium of waterresources technology - Ven te Chow. Univ. of Illinois. 
Ten Cate J.H., Simons J. \& Schreurs H. 1991. - Periphytic macro-algae and micro-algae in dutch freshwater ditches and their significance as ecological indicators of water quality. Arch. Hydrobiol. 122 (3), pp. 275-296.

Univ. de Metz, Laboratoire d'Ecologie (coord.), Ecolor, Gereea, Loisirs et Détente 1991. - Etude des végétaux fixés en relation avec la qualité du milieu. Rapport de contrat Agence de l'Eau Rhin-Meuse, 3 vol. : 94 p + bibliogr ; 64 p annexes ; $\checkmark, 17 \mathrm{p}$ référentiel.
Wiegleb G. 1984. - A study of habitat conditions of the macrophytic vegetation in selected river systems in Western Lower Saxony (Fed. Repub. Ger.). Aquat. Bot., 18, 313-352. Zumstein J.F., Gille E., Décloux J.P., Paris P. \& Picard A. 1989. - Atlas de la lithologie, perméabilité et sensibilité à la pollution des formations affleurantes du Bassin Rhin-Meuse. Agence de l'eau Rhin-Meuse, Moulins-les-Metz, 20 p. 